\title{
Kündigungsfalle Social Media
}

\author{
Isabelle Wildhaber / Silvio Hänsenberger *
}

Die verbreitete Nutzung von sozialen Netzwerken macht auch vor dem Arbeitsplatz nicht halt. Die Vermischung der privaten und der beruflichen Nutzung von Social Media bringt gewisse Risiken mit sich, welche zu einer Kündigung von Seiten des Arbeitgebers führen können. Dieser Beitrag widmet sich den arbeitsrechtlichen Problemen im Zusammenhang mit der Nutzung von Social Media durch den Arbeitnehmer und will aufzeigen, wie mit entsprechenden Fragestellungen umgegangen werden sollte, um alle Beteiligten zu sensibilisieren, aber auch bestehende Risiken zu minimieren.

I. Einleitung .78

II. Verschiedene Konstellationen der Nutzung von Social Media .78

1. Die exzessive Nutzung von Social Media während der Arbeitszeit ...................79

2. Schädliche Äusserungen auf Social Media........................................................ 80

3. Veröffentlichung von Fotos aus dem Arbeitsumfeld auf Social Media .............83

4. Sonstiges Verhalten auf Social Media ...............................................................83

III. Social Media-spezifische Abwägungsgesichtspunkte ............................................85

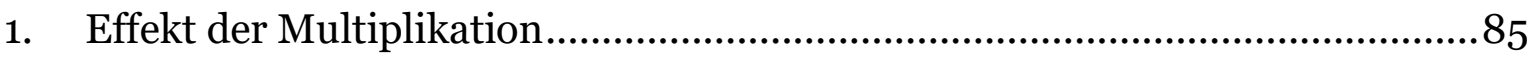

2. Unwissen oder Unsicherheit im Umgang mit Social Media-Plattformen.........85

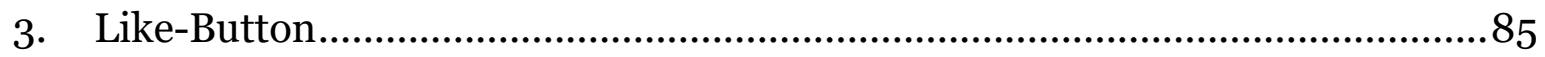

IV. Bemerkungen zu Nachweis und Verwertung im Prozess .......................................86

1. Nutzung eines Accounts durch mehrere Personen..............................................86

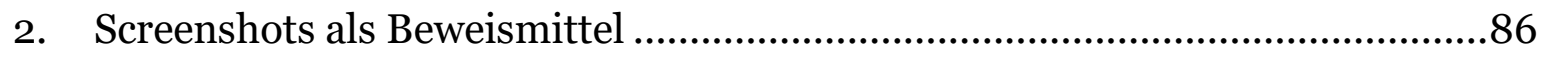

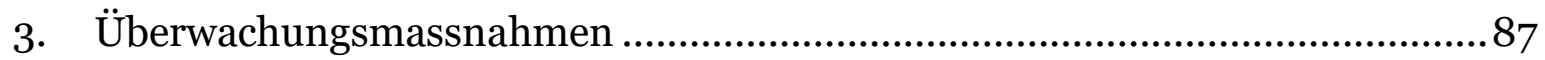

V. Regelung der Social Media-Nutzung durch den Arbeitgeber ................................87

Zitiervorschlag: Isabelle Wildhaber / Silvio Hänsenberger, Kündigungsfalle Social Media, in: sui-generis 2015, S. 77

URL: $\quad$ sui-generis.ch/14

DOI: $\quad$ https://doi.org/10.21257/sg.14

* Prof. Dr. iur., LL.M., Assistenzprofessorin für Arbeits- und Haftpflichtrecht an der Universität St. Gallen; M.A. HSG in law and economics, wissenschaftlicher Assistent.

\footnotetext{
Dieser Beitrag basiert auf einem am 17. April 2015 von der Erstautorin an der Universität St. Gallen gehaltenen Vortrag. Eine ausführliche Version ist in Erscheinung: Isabelle Wildhaber / Silvio Hänsenberger, Kündigung wegen Nutzung von Social Media - wenn Arbeit und Privatleben kollidieren, in: Recht im digitalen Zeitalter, Festgabe Schweizerischer Juristentag 2015 in St. Gallen, Dike Verlag Zürich/St. Gallen 2015, S. 399-430.
} 


\section{Einleitung}

1 Heutzutage ist die Nutzung von Social Media am Arbeitsplatz weit verbreitet. Diese Entwicklung wird durch Umfragen bestätigt. So engagieren sich in der Schweiz $89 \%$ der Grossunternehmen, 83\% der Behörden und Verwaltungen und $59 \%$ der KMU auf Social Media. ${ }^{1}$ Gleichzeitig zeigen Studien, dass mehr als ein Drittel der Arbeitnehmer in der Schweiz täglich während der Arbeitszeit in Facebook oder auf anderen sozialen Netzwerken aktiv ist. ${ }^{2}$

2 Diese allgegenwärtige Nutzung führt zum Risiko des Missbrauchs. Die feine Linie zwischen der beruflichen und der privaten Sphäre bei der Nutzung von Social Media verschwimmt immer mehr. 3 Wer lästert und schimpft nicht gern einmal über seinen Arbeitgeber? In sozialen Netzwerken sollte man damit lieber vorsichtig sein. Sonst könnte eine Kündigung drohen.

3 Welche Aktivitäten können nun dazu führen, dass der Arbeitgeber kündigt? Schweizerische Rechtsprechung in diesem Bereich gibt es kaum oder sie ist nicht veröffentlicht. 4 Branchenübergrei-

\footnotetext{
ZHAW, Studie Social Media Schweiz 2013.

XEIT GmbH, Social Media Studie 2014.

Daniel Alder, E-Mail-Daten am Arbeitsplatz im Fokus von Datenschutz- und Arbeitsrecht, Revue de l'avocat 6/7/2013, S. 276 ff., S. 277; Jean-Philippe Dunand, Internet au travail: droits et obligations de l'employeur et du travailleur, in: JeanPhilippe Dunand/Pascal Mahon (Hrsg.), Internet au travail, Genf 2014, S. 33 ff., S. 39; Carole Aubert/Régine Delley, Utilisation des réseaux sociaux par les travailleurs et les employeurs, in: JeanPhilippe Dunand/Pascal Mahon (Hrsg.), Internet au travail, Genf 2014, S. 133 ff.; Wolfgang Däubler, Entgrenzung der Arbeit - Ein Problem des Arbeitsrechts?, Soziales Recht 2/2014, S. 45 ff.

4 Siehe dazu Daniel Hürlimann, Publikation von Urteilen durch Gerichte, in: sui-generis 2014, S. 82.
}

fende Kodizes fehlen.5 Ebenso wenig existieren gesetzliche Spezialregelungen. Der Bundesrat lehnte es im Oktober 2013 $\mathrm{ab}$, ein spezielles Gesetz für Social Media vorzulegen. ${ }^{6}$ Es kommen deshalb grundsätzlich die allgemeinen Gesetzesbestimmungen zur arbeitsrechtlichen Kündigung, das sind Art. 335 ff. OR, zur Anwendung, es sei denn, ein anwendbarer GAV bestimmte etwas anderes.

4 Dieser Beitrag hat zum Ziel, verschiedene Konstellationen der Nutzung von Social Media zu analysieren, um festzustellen, ob diese zu einer ordentlichen oder fristlosen Kündigung führen könnten (nachfolgend II.). Sodann sollen einige SocialMedia-spezifische Abwägungsgesichtspunkte erläutert werden (nachfolgend III.), Bemerkungen zu Nachweis und Verwertung im Prozess gemacht werden (nachfolgend IV.), gefolgt von der Empfehlung einer Regelung durch den Arbeitgeber (nachfolgend V.).

\section{Verschiedene Konstellationen der Nutzung von Social Media}

5 Grundsätzlich gilt in der Schweiz die Kündigungsfreiheit gemäss Art. 335 Abs. 1 OR.7 Es braucht keinen besonderen Grund, um ordentlich kündigen zu können. Es gibt aber bestimmte Gründe oder Motive, derentwegen nicht gekündigt werden darf, weil ansonsten die Kündigung gemäss Art. 336 OR missbräuchlich ist. 8

5 Stefan Mair, Schweigen oder fliegen, Handelszeitung vom 16. Januar 2015.

6 Bundesrat, Rechtliche Basis für Social Media, Stellungnahme des Bundesrates zum Postulat Amherd 11.3912 vom 29.9.2011.

7 BGE 132 III 115 E. 2.1; BGE 131 III 535 E. 4.1.

8 Wobei die Aufzählung von Art. 336 nicht abschliessend ist, s. dazu BGE 136 III 513

E. 2.1; BGE 132 III 115 E. 2.1. 
Die Kündigung kann insbesondere missbräuchlich sein, weil der Arbeitnehmer ein verfassungsmässiges Recht im Sinne von Art. 336 Abs. 1 lit. b OR ausübt. In unserem Kontext wäre das die Meinungsäusserungsfreiheit. Die Kündigung ist aber nach Art. 336 Abs. 1 lit. b in fine OR nicht missbräuchlich, wenn die Meinungsäusserung eine Pflicht aus dem Arbeitsverhältnis verletzt oder die Zusammenarbeit im Betrieb wesentlich beeinträchtigt. 9 Es muss also abgewogen werden zwischen der Meinungsäusserungsfreiheit des Arbeitnehmers und anderen Pflichten und Interessen.

7 Als härtere Massnahme kommt die fristlose Kündigung des Arbeitnehmers in Frage, wenn dem Kündigenden nach Treu und Glauben die Fortsetzung des Arbeitsverhältnisses bis zum Ablauf der ordentlichen Kündigungsfrist nicht mehr zugemutet werden kann (Art. 337 Abs. 1 und Abs. 2 OR). Hier kann mitberücksichtigt werden, ob der Arbeitgeber klare Weisungen zur Benutzung von Social Media erlassen hat, ob er sich früher bereits eindeutig gegen die missbräuchliche Nutzung von Social Media oder ob er sogar diesbezügliche Verweise oder Verwarnungen $^{10}$ ausgesprochen hat. Nach derzeitiger Schweizer Rechtsprechung

BGE 130 III 699 E. 4; Adrian Staehelin, Der Arbeitsvertrag. Art. 330b-355 OR, Art. 361-362 OR, in: Kommentar zum schweizerischen Zivilgesetzbuch (Zürcher Kommentar), Bd. V/2c, Jörg Schmid (Hrsg.), 4. Aufl., Zürich 2014, Art. $336 \mathrm{~N}$ 20 f.; Wolfgang Portmann, Basler Kommentar, Obligationenrecht I, Art. 1 529, Heinrich Honsell/Nedim Peter Vogt/Wolfgang Wieland (Hrsg.), 5. Aufl., Basel 2011, Art. 336 N 9.

10 Übersicht zur Rechtsprechung zur Verwarnung BGer 4C.364/2005 vom 12.1.2006, E. 2; differenziert zur Notwendigkeit einer vorgängigen Verwarnung Ullin Streiff/Adrian von Kaenel/Roger Rudolph, Arbeitsvertrag. Praxiskommentar zu Art. 319-362 OR, 7. Aufl., Zürich 2012, Art. 337 $\mathrm{N}$ 13, m.w.H. wird die Schwelle für eine fristlose Entlassung hoch angesetzt. ${ }^{11}$

\section{Die exzessive Nutzung von Social Media während der Arbeitszeit}

Fallbeispiel 1: In einem Fall der Personalrekurskommission BS vom 31. August 2004 besuchte der Ressortleiter einer kantonalen Dienststelle über mehrere Monate täglich 2-3 Stunden am Tag Sex-Seiten. Die Dauer der täglichen Nutzung sowie der Verstoss gegen eine Weisung, die den Konsum pornografischer Inhalte verbot, hatten eine fristlose Kündigung zur Folge. Diese befand die Personalrekurskommission als rechtens. ${ }^{12}$

8 Bei der exzessiven Nutzung von Social Media während der Arbeitszeit ist zwischen der dienstlichen und der privaten Nutzung zu unterscheiden. Die dienstliche Nutzung ist Teil der Arbeitsleistung durch den Arbeitnehmer. Es kommt dabei darauf an, ob die Aktivität des Arbeitnehmers in sozialen Netzwerken privat oder dienstlich veranlasst ist. 13 Bei einer geschäftlichen Nutzung muss stets ein Bezug zu den geschäftlichen Aufgaben bestehen. ${ }^{14}$

11 BGer 4C.463/1999 vom 4.7.2000, E. 9 f.; BGer 4C.106/2001 vom 14.2.2002, E. 3c; BGer 4C.349/2002 vom 25.6.2003, E. 5; BGer4C.109/2003 vom 30.7.2003, E. 2; BGer 4C.173/2003 vom 21.10.2003, E. 3.2.

12 Entscheid der Personalrekurskommission BS vom 31.08.2004.

13 Andreas Borsutzky, Soziale Netzwerke - Regelungskompetenz des Arbeitgebers und Mitbestimmungsrechte des Betriebsrats, NZA 2013, S. $647 \mathrm{ff}$.

14 Christoph Holenstein, Die Benutzung von elektronischen Kommunikationsmitteln (Internet und Intranet) im Arbeitsverhältnis, Diss. Bern 2002, S. $71 \mathrm{ff}$. 
9 Mit der privaten Nutzung von Social Media während der Arbeitszeit kann der Arbeitnehmer grundsätzlich seine Pflicht zur Leistung von Arbeit nach Art. 319 OR verletzen. Es kann auch eine Verletzung der Treuepflicht des Arbeitnehmers gemäss Art. 321a OR vorliegen. ${ }^{15}$

Bei der privaten Nutzung gilt deshalb Folgendes: Fehlt eine Anweisung des Arbeitgebers zur privaten Nutzung des Internets und Social Media, so ist die Privatnutzung grundsätzlich zulässig, solange die Arbeitsleistung nicht gefährdet ist und das übliche Mass nicht überschritten wird. ${ }^{16}$ Alles in allem kann die exzessive Nutzung des Internets während der Arbeitszeit $\mathrm{zu}$ privaten Zwecken eine schwere Pflichtverletzung des Arbeitsvertrages darstellen, die den Arbeitgeber ohne vorangegangene Verwarnung zu einer ordentlichen oder gar fristlosen Kündigung des Arbeitsverhältnisses berechtigen kann. ${ }^{17}$ Dennoch verbleibt ein gros-

15 Dunand (Fn. 3), S. 33 ff., 36 ff., $41 \mathrm{f}$.

16 Urs Egli, Soziale Netzwerke und Arbeitsverhältnisse, Jusletter 17. Januar 2011, Rz. 36, 42, 48; Aubert/Delley (Fn. 3), S. 154; Holenstein (Fn. 14), S. 47, S. 82; Streiff/von Kaenel/Rudolph (Fn. 10), Art. 328b N 17; Bruno Baeriswyl, in: Aktuelle Probleme des Arbeitsrechts, Zürich 2005, 10 Jahre Datenschutz im Arbeitsrecht, S. 62; Beat Rudin, Was darf die Chefin, was die Angestellte? Arbeits- und datenschutzrechtliche Schranken der technischen Überwachung der Internet-Nutzung am Arbeitsplatz, DIGMA 2001, S. 4 ff.; Gabriel Aubert, Art. 319-362 CO, in: Commentaire romand Code des obligations I, Luc Thévenoz/ Franz Werro (Hrsg.), 2. Aufl., Basel 2012, Art. $328 b$ N 8.

17 Vgl. Dunand (Fn. 3), S. 41 f., m.w.H.; BAG, Urteil vom 7.7.2005, 2 AZR 581/04; LAG RheinlandPfalz, Urteil vom 06.02.2012, 6 Sa 682/09) Vgl. auch LAG Niedersachsen, Urteil vom 31.5.2010, 12 Sa 875/o9 (exzessive Privatnutzung des Dienst-PC, wenn der Arbeitnehmer über einen Zeitraum von mehr als sieben Wochen arbeitstäglich mehrere Stunden mit dem Schreiben und Beantworten privater E-Mails verbringt, an mehreren Tagen sogar in einem zeitlichen Umfang, der gar keinen Raum für die Erledigungen von ser Unschärfebereich. Klarheit kann ein Nutzungsreglement schaffen (nachfolgend V.).

\section{Schädliche Äusserungen auf Social Media}

11 Arbeitnehmer können schädliche Äusserungen über den Arbeitgeber, ${ }^{18}$ über Vorgesetzte, ${ }^{19}$ über Arbeitskollegen,20 über Geschäftspartner des Arbeitgebers oder über Kunden des Arbeitgebers machen, die eine fristlose oder ordentliche Kündigung nach sich ziehen können.

Fallbeispiel 2: Der damals 27-jährige Hubert F. bezeichnete seinen Arbeitgeber auf Facebook als «Menschenschinder» und «Ausbeuter» und schrieb über seinen Job, dass er «dämliche Scheisse für Mindestlohn minus 20\%» erledigen müsse. Das Landesarbeitsgericht Hamm entschied, dieser Eintrag stelle eine massiv ehrverletzende Äusserung dar. Er berechtige den Arbeitgeber zur fristlosen Kündigung. ${ }^{21}$

Fallbeispiel 3: Eine Arbeitnehmerin hatte auf ihrer Facebook-Chronik über die mangelhafte Abwicklung ihres privaten Handyvertrages bei $\mathrm{O} 2$ gelästert: «Boah kotzen die mich an

Dienstaufgaben mehr lässt).

18 ArbG Iserlohn, Urteil vom 9.3.2010, $5 \mathrm{Ca}$ 2640/o9; LAG Baden-Württemberg vom 10.2.2010, 2 Sa 59/o9 (Blogbeitrag eines Arbeitnehmers, der dem Arbeitgeber «eine verschärfte Ausbeutung, Angriffe auf politische und gewerkschaftliche Rechte sowie menschenverachtende Jagd auf Kranke» vorwarf, noch von dem Recht auf freie Meinungsäusserung gedeckt).

19 ArbG Hagen, Urteil vom 16.5.2012, 3 Ca 2597/11.

20 ArbG Duisburg, Urteil vom 26.9.2012, 5 Ca 949/12.

21 LAG Hamm, Urteil vom 10.10.2012, 3 Sa 644/12. Vgl. aber ArbG Bochum, Urteil vom 9.2.2012, 3 Ca 1203/11. 
von O2, da sperren sie einfach das Handy, obwohl man schon bezahlt hat ... und dann behaupten die es wären keine Zahlungen da. Solche Penner ... Naja ab nächsten Monat habe ich einen neuen Anbieter ...». Leider war die Arbeitnehmerin gerade im Auftrag ihres Arbeitgebers bei $\mathrm{O} 2$ eingesetzt. $\mathrm{O} 2$ beschwerte sich beim Arbeitgeber über diesen «diffamierenden» Eintrag. Der Bayerische Verwaltungsgerichtshof urteilte, dass solch ein Beitrag keine fristlose Kündigung rechtfertige. Die Arbeitnehmerin habe in ihrer Eigenschaft als Kundin und nicht als Arbeitnehmerin agiert. ${ }^{22}$

Die konkrete Frage, die sich stellt, ist stets, was der Arbeitnehmer sagen darf und was nicht. Die Meinungsäusserungsfreiheit nach Art. 16 Abs. 2 und 3 BV 23 wird durch Art. 336 Abs. 1 lit. b OR konkretisiert. ${ }^{24}$ Dieser besagt wie erwähnt, dass eine Kündigung wegen einer Meinungsäusserung des Arbeitnehmers missbräuchlich ist, es sei denn, diese verletze eine Pflicht aus dem Arbeitsverhältnis oder beeinträchtige die Zusammen-

22 Bayrischer VGH, Urteil vom 29.2.2012, 12 C 12.26. Vgl. auch LAG Schleswig-Holstein vom 8.4.2010, 4 Sa 474/09 (Bezeichnung eines Kunden als «Arschloch»). S. dazu auch Michael Kort, Kündigungsrechtliche Fragen bei Äusserungen des Arbeitnehmers im Internet, NZA 2012, S. 1321 ff., 1322.

23 Ulrich Häfelin/Walter Haller/Helen Keller, Schweizerisches Bundesstaatsrecht, 8. Aufl., Zürich 2012, Rz. 456 f.

24 Günther Wiese, Internet und Meinungsfreiheit des Arbeitgebers, Arbeitnehmers und Betriebsrats, NZA 2012, S. 1 ff., 4; Kort (Fn. 22), S. 1321. Das Recht, gegenüber dem Arbeitgeber auf Missstände hinzuweisen, ergibt sich nach neuerer Rechtsprechung nicht aus der Meinungsäusserungsfreiheit, sondern aus den gegenseitigen Fürsorge- und Treuepflichten (BGer 4A_432/2009 vom 10.11.2009, E. 2.2.2). arbeit im Betrieb wesentlich. ${ }^{25}$

13 Die Meinungsäusserungsfreiheit hat also Grenzen. Auch eine ansonsten zulässige Kritik kann sich wegen der Öffentlichkeit der Äusserung in einem sozialen Netzwerk als unzulässiger Verstoss gegen die vertragliche Treuepflicht darstellen. ${ }^{26}$ Grenze der Meinungsäusserungsfreiheit ist ausserdem der Schutz der persönlichen Verhältnisse und der Ehre anderer Personen. ${ }^{27}$

14 Es ist dem Arbeitnehmer grundsätzlich nicht verwehrt, sich kritisch über seine Arbeit, sein Arbeitsverhältnis, den Arbeitgeber oder dessen Kunden zu äussern, ${ }^{28}$ unter Umständen auch überspitzt. Aber grob schmähende Angriffe, Beleidigungen oder Lügen muss ein Arbeitgeber nicht hinnehmen.

15 Noch ganz am Anfang steht die schweizerische Lehre und Rechtsprechung zur Frage, ob und wann die beleidigende Äusserung auf Social Media wegen ihres privaten Charakters besonderen Schutz

25 Wolfgang Portmann, Basler Kommentar, Obligationenrecht I, Art. 1-529, Heinrich Honsell/Nedim Peter Vogt/Wolfgang Wieland (Hrsg.), 5. Aufl., Basel 2011, Art. 336 N 9.

26 Vgl. Streiff/von Kaenel/Rudolph (Fn. 10), Art. 336 N 6, m.w.H.

27 Ulrich Häfelin/Walter Haller/Helen Keller (Fn. 23), Rz. 484; Andreas Kley/Esther Tophinke, Art. 16, in: Die Schweizerische Bundesverfassung Kommentar, Bernhard Ehrenzeller/Philippe Mastronardi/Rainer J. Schweizer/Klaus A. Vallender (Hrsg.), 3. Aufl., St. Gallen 2014, Art. 16 N 14.

28 Christiane Brunner/Jean-Michel Bühler/ JeanBernard Waeber/Christian Bruchez, Kommentar zum Arbeitsvertragsrecht, 3. Aufl., Basel 2005, N 5; Martin Farner, Missbräuchliche Kündigung (Art. 336 Abs. 1 Bst. a OR), Kritik am Vorgesetzten, Bemerkungen zum Urteil des Bundesgerichts, I. Zivilabteilung, 19.12.2000, M. c. R., Berufung, in: AJP 2001, S. 710 ff.; Martin Kock, Unmutsäusserungen und Beleidigungen auf Facebook \& Co. als Kündigungsgrund, DB 2013, S. 934 ff. 
geniesst. Bei vertraulichen Gesprächen im privaten Rahmen, also z.B. beim Kaffeetratsch oder am Stammtisch, darf der Arbeitnehmer regelmässig darauf vertrauen, dass seine Äusserungen nicht nach aussen getragen werden. Tatsächlich wird es für die Wirksamkeit einer Kündigung im Einzelfall darauf ankommen, auf welchem Kommunikationskanal und vor welchem Publikum der Arbeitnehmer sich geäussert hat. ${ }^{29}$

Somit kommt der Unterscheidung zwischen öffentlicher und privater Äusserung eine entscheidende Bedeutung zu. Der Begriff der Öffentlichkeit wird im Schweizer Recht nicht einheitlich ver- wendet. $3^{\circ}$ Öffentlich ist, was nicht im privaten Umfeld erfolgt. Im vorliegenden Kontext kann als privater Rahmen ein begrenzter Kreis an Beteiligten, welche durch eine persönliche Beziehung oder ein besonderes Vertrauen miteinander verbunden sind, definiert werden. ${ }^{31}$

17 Die folgende Grafik zeigt, welche Kanäle u.E. als privat und welche als öffentlich einzuschätzen sind. Als Leitlinie kann man bildlich von einer Handvoll Freunde sprechen..$^{2}$ Alles andere ist - wie die Grafik zeigt - in unseren Augen öffentlich.

\begin{tabular}{|c|c|c|}
\hline Publikationsort innerhalb eines Sozialen Netzwerkes & öffentlich & privat \\
\hline 1. Im öffentlichen Profil & $\mathrm{X}$ & \\
\hline 2. Im Profil für Freunde plus Freunde von Freunden & $\mathrm{X}$ & \\
\hline $\begin{array}{l}\text { 3. In einem Posting in der eigenen Chronik oder in einer «geschlossenen» bzw. } \\
\text { «geheimen» Gruppe, welche für sechs oder mehr Freunde zugänglich ist } 33\end{array}$ & $\mathrm{X}$ & \\
\hline $\begin{array}{l}\text { 4. In einem Posting in der eigenen Chronik oder in einer «geschlossenen» } \\
\text { bzw. «geheimen» Gruppe, welches für weniger als sechs Freunde zugäng- } \\
\text { lich ist, falls die Freunde zum Erklärenden in einer besonderen persönli- } \\
\text { chen oder beruflichen Nähebeziehung stehen und der Anlass der Kommu- } \\
\text { nikation privater Natur ist } 34\end{array}$ & & $\mathrm{X}$ \\
\hline 5. In einem Posting in einer fremder Chronik 35 & $\mathrm{X}$ & \\
\hline 6. In einer «offenen» Facebook-Gruppe ${ }^{36}$ & $\mathrm{X}$ & \\
\hline $\begin{array}{l}\text { 7. In einer Facebook-Nachricht/Chat, }{ }^{37} \text { sofern weniger als sechs Personen } \\
\text { daran teilnehmen und die Voraussetzungen gem. Ziff. } 4 \text { erfüllt sind }\end{array}$ & & $\mathrm{X}$ \\
\hline
\end{tabular}

29 Jobst-Hubertus Bauer/Jens Günther, Kündigung wegen beleidigender Äusserungen auf Facebook vertrauliche Kommunikation unter Freunden?, NZA 2013, S. 67 ff., 68; vgl. auch Aubert/Delley (Fn. 3), S. 159 f., m.w.H.

30 So unterscheiden sich z.B. die Öffentlichkeitsbegriffe von Art. 259 ff. StGB, Art. 652a OR, Art. 54 ZPO und Art. 19 Abs. 1 Bst. a URG.

${ }_{31}$ Vgl. BGE 130 IV 111 E. 5.2 im Zusammenhang mit dem Rassismusartikel (Art. 261 ${ }^{\text {bis }} \mathrm{StGB}$ ). Zum Begriff der Öffentlichkeit siehe Marcel A. Niggli,

\begin{abstract}
Rassendiskriminierung - Ein Kommentar zu Art. $261^{\text {bis }}$ StGB und Art. 171c MStG mit Rücksicht auf das «Übereinkommen vom 21. Dezember 1965 zur Beseitigung jeder Form von Rassendiskriminierung» und die entsprechenden Regelungen anderer Unterzeichnerstaaten, 2. Aufl., Zürich 2007, S. 297 ff. In BGer 6B_256/2014 vom 8.4.2015 (FacebookPost: Freundeskreis ist nicht «Bevölkerung») musste das Bundesgericht die Frage, ob eine Äusserung gegenüber Facebook-Freunden als «öffentlich» oder «privat» zu gelten hat, nicht entscheiden. Der Be-
\end{abstract}




\section{Veröffentlichung von Fotos aus dem Arbeitsumfeld auf Social Me- dia}

Fallbeispiel 4: Eine BundeshausAngestellte veröffentlichte wiederholt Nackt-Selfies und pornographische Videos, die sie von sich selbst in den Räumlichkeiten des Bundeshauses aufgenommen hatte. Sie tat dies auf ihrem Twitter-Account. Sie hatte 11'ooo Follower. ${ }^{3} 8$

18 Veröffentlicht ein Arbeitnehmer auf seinem privaten Account innerhalb eines sozialen Netzwerks Bilder und stehen diese im Zusammenhang mit seiner beruflichen Tätigkeit, kann dies zu einem Konflikt mit seinem bestehenden Arbeitsverhältnis und $\mathrm{zu}$ einer Verletzung der arbeitsvertraglichen Treuepflichten (Art. 321a OR) führen39.

Folgende Kriterien werden relevant sein:

griff der «Bevölkerung» ist nicht gleichbedeutend mit demjenigen der «Öffentlichkeit», der unter anderem beim Tatbestand der Rassendiskriminierung massgebend ist.

32 Antje Burmester, Die «Facebook-Kündigung» im Spiegel der aktuellen Rechtsprechung, ArbRB 2013, S. 245 ff.

33 Bauer/Günther (Fn. 29), S. 69 f.; Kock (Fn. 28), S. 934 ff., S. 937; ArbG Hagen, Urteil vom 16.5.2012, 3 Ca 2597/11; ArbG Dessau-Rosslau, Urteil vom 21.2.2012, 1 Ca 148/11.

34 Kock (Fn. 28), S. 938; Bauer/Günther (Fn. 29), S. 70.

35 Bauer/Günther (Fn. 29), S. 70.

36 Bauer/Günther (Fn. 29), S. 70.

37 Kock (Fn. 28), S. 937; Bauer/Günther (Fn. 29), S. 70; Lars Hinrichs/Martin Hörtz, Web 2.0: Bild‘ Dir Deine Meinung - auf Kosten des Arbeitgebers, NJW 2013, S. 648 ff., S. 652.

38 NZZ vom 6.8.2014, Nackt-Selfies aus dem Bundeshaus.

39 Bei vorstehendem Fallbeispiel 4 leitet sich die Treuepflicht der Bundeshaus-Angestellten aus Art. 20 Abs. 1 BPG (SR 172.220.1) ab.
- Sind auf den Fotos Personen abgebildet, deren allgemeines Persönlichkeits- und/ oder Urheberrecht durch die Veröffentlichung beeinträchtigt wird? Können sie identifiziert werden?

- Kann ein Zusammenhang mit dem Unternehmen des Arbeitgebers hergestellt werden?

- Ist das Foto ehrverletzend oder rufschädigend für den Arbeitgeber? Dies ist oft eine Wertungsfrage. Eine Rolle spielen kann dabei auch, ob der Arbeitnehmer eine leitende oder sonstwie besondere Funktion innehatte.

- Ebenso wird im Einzelfall danach zu fragen sein, ob der Arbeitnehmer durch die Veröffentlichung gegen besondere vertragliche oder gesetzliche Schweigepflichten verstossen hat, was insbesondere in sensiblen Tätigkeitsfeldern naheliegt (z.B. Gesundheitswesen, Rechtsberatung, Bankwesen).

- Letztlich ist auch zu berücksichtigen, wie gross der Personenkreis ist, der auf das hochgeladene Foto Zugriff hat.

\section{Sonstiges Verhalten auf Social Media}

2o Über die Fallgruppen beleidigender Äusserungen und der Veröffentlichung von Fotos aus dem Arbeitsumfeld hinaus ist eine Vielzahl weiterer Verhaltensweisen des Arbeitnehmers in sozialen Netzwerken denkbar, die den Arbeitgeber zum Aussprechen einer fristlosen Kündigung berechtigen können.

21 Erstens kann das Verhalten des Arbeitnehmers in sozialen Netzwerken in den Zeiten, in denen dieser (vermeintlich) arbeitsunfähig erkrankt ist, eine arbeitsvertragliche Pflichtverletzung darstellen oder eine solche aufdecken. Der Arbeit- 
geber kann sich fragen, ob die Arbeitsunfähigkeit vielleicht nur vorgetäuscht ist. Dies kann eine ordentliche oder fristlose Kündigung je nachdem rechtfertigen. ${ }^{40}$

Fallbeispiel 5: Am 13. November 2008 war eine 31-jährige Baslerin mit einer starken Migräne für einen Tag zu Hause geblieben. Eine Woche später wurde ihr von der Versicherung Nationale Suisse fristlos gekündigt, weil die Arbeitnehmerin angegeben hatte, bei Migräne nicht am Bildschirm arbeiten zu können, sie aber trotzdem etliche Zeit auf Facebook war. ${ }^{41}$

Zweitens kann die Veröffentlichung kompromittierender Privatfotos des Arbeitnehmers eine Kündigung nach sich ziehen. Wenn der Arbeitnehmer in seinem Netzwerk-Profil unter der Rubrik «Arbeitgeber» das Unternehmen angegeben hat, er über eine herausgehobene Stellung innerhalb des Unternehmens verfügt oder eine Vielzahl von NetzwerkFreundschaften mit Kunden bzw. Geschäftspartnern seines Arbeitgebers pflegt, kann das eine Kündigung rechtfertigen. ${ }^{42}$

40 Eine fristlose Kündigung war gerechtfertigt bei Arbeit für einen Dritten während der Krankheit (BGer in JAR 1999 S. 289, GSG BS in JAR 1990 S. 251), ebenfalls nachdem ein «arbeitsunfähiger» Maurer bei sich zuhause Maurerarbeiten ausführte (BGer 4A_582/2010 vom 28.1.2011); für nicht gerechtfertigt taxiert wurde die fristlose Kündigung eines Arbeitnehmers, der, während er krankgeschrieben war, zweimal zur Jagd ging (Urteil BVGer A-73/2014 vom 14.7.2014, E. 4.5).

41 S. den Sachverhalt in 20 Minuten Online vom 23.4.2009, Facebook besucht, Kündigung erhalten.

42 Vgl. ArbG Hamburg, Urteil vom 18.9.2013, $27 \mathrm{Ca}$ 207/13 (Ein Polizist hatte ein Foto auf seine private Facebook-Seite hochgeladen, welches einen Totenkopf mit einer Polizeimütze vor einer jüdischen Schule zeigte).
23 Drittens dürften den Nationalsozialismus verherrlichende oder andere rassistische, extremistische, religionsverachtende oder diskriminierende Einträge in sozialen Medien grundsätzlich geeignet sein, den Arbeitgeber in schwerwiegenden Fällen $\mathrm{zu}$ einer fristlosen Kündigung zu veranlassen. 43

Fallbeispiel 6: Ein ehemaliger Zürcher SVP-Lokalpolitiker twitterte im Juni 2012: «Vielleicht brauchen wir wieder eine Kristallnacht ... diesmal für Moscheen». Sein Arbeitgeber hatte ihm daraufhin fristlos gekündigt. 44

24 Viertens kann eine Kündigung wegen des Verrats von Geschäfts- und Betriebsgeheimnissen auf Social Media erfolgen. Das Geschäfts- und Betriebsgeheimnis folgt auch ohne ausdrückliche Vereinbarung aus der arbeitsvertraglichen Treuepflicht in Art. 321a Abs. 4 OR. Bei der Beteiligung von Mitarbeitern an «Wikis», «Blogs» oder Social Media besteht das Risiko, dass der Arbeitnehmer relevante Interna des Unternehmens oder sogar Geschäfts- und/oder Betriebsgeheimnisse preisgibt.

25 Dies gilt namentlich für fachbezogene Foren, die dem Austausch von Wissen dienen. Ist dieser Wissenstransfer der Tätigkeit des Arbeitnehmers und damit zugleich auch dem Unternehmen zuzuordnen, bedarf es einer besonderen Sen-

43 Zur fristlosen Kündigung wegen Delikten des Arbeitnehmers s. Streiff/von Kaenel/Rudolph (Fn. 10), Art. 337 N 5, m.w.H.

44 S. dazu auch Marcel Gyr, Arbeitgeber trennt sich von SVP-Twitterer, NZZ Online, 27.06.2012; Brigitte Hürlimann, Der «Kristallnacht-Twitterer» darf namentlich genannt werden, NZZ Nr. 81, 9.4.2015; Martin Steiger, Urteil: Twitter-Nutzer $=$ Relative Person der Zeitgeschichte. 
sibilität, da dies Rückschlüsse auf das Forschungs- und Geschäftsverhalten des Unternehmens zulässt. Insbesondere der unzulässige Austausch über Kundendaten oder wirtschaftlich sensible Informationen wie Preispolitik oder Marktstrategien mittels der sozialen Medien hat erhebliche Auswirkungen für ein Unternehmen. 45

\section{Social Media-spezifische Abwä- gungsgesichtspunkte}

\section{Effekt der Multiplikation}

26 Die hohe Verbreitungsgeschwindigkeit von Äusserungen im Internet ist zu berücksichtigen. Äusserungen eines Nutzers in sozialen Netzwerken können sich unkontrolliert schnell und weit verbreiten. ${ }^{6}$ Eine rufschädigende Wirkung kann wesentlich schneller und in weitaus grösserem Umfang eintreten, als dies auf klassischen Kommunikationswegen der Fall wäre.47 Das Risiko der Störung des Betriebsfriedens und der Vertrauensbeziehung zum Arbeitgeber ist ungleich höher als bei mündlichen Äusserungen im vertrauten Gespräch. Diese spezifischen Umstände der Verbreitungsgeschwindigkeit eines Social Media-Eintrages dürften im Rahmen der Interessenabwägung im Regelfall zu Lasten des Arbeitnehmers gewertet werden. 48

45 Philipp Byers/Stefan Mössner, Die Nutzung des Web 2.0 am Arbeitsplatz: Fluch und Segen für den Arbeitgeber, BB 2012, S. 1665 ff., S. 1666.

46 Alexander Bissels/Martin Lützeler/Gerlind Wisskirchen, Facebook, Twitter \& Co.: Das Web 2.0 als arbeitsrechtliches Problem, BB 2010, S. 2433 ff.

47 Dominique Perron/Grégory Jouk, Risque réputationnel - les réseaux sociaux changent-ils la donne?, Revue de l'expert comptable suisse 9/2012, S. $624 \mathrm{f}$.

$48 \mathrm{Im}$ Ergebnis ebenso Christian Oberwetter, Soziale Netzwerke im Fadenkreuz des Arbeitsrechts, NJW
27 Man spricht vom sogenannten Effekt der Multiplikation. Der vorhin erwähnte SVP-Twitterer hatte auf Twitter weniger als 400 Follower, die seine Meldungen direkt abonniert hatten. Durch ReTweets waren aber innert Kürze 40'0oo Menschen erreicht worden. Da half es auch nichts, dass er den Original-Tweet schon fünf Minuten nach Versendung wieder vom Netz nahm. 49 Vielleicht gilt es, ab und zu das alte Sprichwort zu beherzigen «Reden ist Silber, Schweigen ist Gold», oder modern: «Twittern ist Silber, Schweigen ist Gold.»

\section{Unwissen oder Unsicherheit im Umgang mit Social Media- Plattformen}

28 Auch die Unwissenheit oder Unsicherheit im Umgang mit der Social MediaPlattform kann nur ausnahmsweise zu Gunsten des Arbeitnehmers in die Interessenabwägung einfliessen. Wer eine Social Media-Plattform wie Facebook als «Kommunikationskanal» für seine Äusserung wählt, nimmt zumindest eventualvorsätzlich in Kauf, dass diese einem grösseren als eigentlich beabsichtigten Kreis zur Kenntnis gelangt, falls er mit der Bedienung dieses Mediums nicht hinreichend vertraut ist. 50

\section{Like-Button}

29 Eine weitere interessante Frage ist, inwieweit sich Arbeitnehmer durch das Drücken des «Gefällt mir»-Button («Li-

2011, S. 417 ff., S. 420; Bissels/Lützeler/Wisskirchen (Fn. 46), S. 2435.

49 Marcel Gyr, Arbeitgeber trennt sich von SVPTwitterer, NZZ Online, 27.06.2012. S. auch Arno Frings/Ulrich Wahlers, Social Media, IPad \& Co. im Arbeitsverhältnis, BB 2011, S. 3126 ff.; Byers/Mössner (Fn. 45), S. 1665.

50 ArbG Hagen, Urteil vom 16.05.2012, 3 Ca 2597/11. 
ken») eine (rechtswidrige) Aussage zu Eigen machen und damit ebenfalls dafür verantwortlich gemacht werden können. Wiegt das weniger schwer als der Original-Eintrag?51

3o Grundsätzlich kann das Drücken des «Gefällt mir»-Buttons durchaus zu einer «Vereinnahmung» der Äusserung und damit zur Verantwortlichkeit des betreffenden Arbeitnehmers führen. In dieser Verhaltensweise kann genauso eine Treuepflichtverletzung gegenüber dem Arbeitgeber gesehen werden wie bei einer selbst verfassten beleidigenden Äusserung. ${ }^{2}$ Derjenige, der durch die Aktivierung des «Gefällt mir»-Buttons seine Zustimmung zu der beleidigenden Äusserung erklärt, macht dies für andere Nutzer deutlich sichtbar und sorgt dabei auch für eine Verbreitung und Wiederholung der schädigenden Äusserung. Dem «Like» kann also der «Dislike» folgen in Form der Kündigung.

\section{Bemerkungen zu Nachweis und Verwertung im Prozess}

31 Ficht der Arbeitnehmer eine Kündigung an, so trägt der Arbeitgeber die Darlegungs- und Beweislast für die arbeitsvertragliche Pflichtverletzung des Arbeitnehmers.53 Dabei kann der Arbeitgeber folgende drei Probleme haben.

51 So ArbG Dessau-Rosslau, Urteil vom 21.2.2012, 1 Ca 148/11 (die Betätigung des Buttons spreche in der Regel für eine spontane Reaktion ohne nähere Überlegung, die in ihrem Bedeutungsgehalt nicht zu hoch eingeschätzt werden dürfe).

52 Bauer/Günther (Fn. 29), S. 71, m.w.H.; ArbG Dessau-Rosslau, Urteil vom 21.2.2012, 1 Ca 148/11.

53 Holenstein (Fn. 14), S. 164 ff.

\section{Nutzung eines Accounts durch mehrere Personen}

32 Bei zugangsbeschränkten Social Media bestehen dann Beweisschwierigkeiten, wenn der Account des Arbeitnehmers von mehreren Personen genutzt wird. Der Arbeitnehmer kann im Prozess behaupten, der betreffende Inhalt sei nicht von ihm selbst, sondern von einem Dritten (z.B. dem Ehepartner) eingestellt worden.54 Grundsätzlich dürfte jedoch ein dringender Verdacht dafür sprechen, dass der jeweilige Inhaber des Profils auch Urheber der veröffentlichten Inhalte ist. Um seine eigene Urheberschaft und Verantwortlichkeit zu widerlegen, muss der Arbeitnehmer u.E. detaillierte Angaben zu seiner fehlenden Täterschaft machen, d.h. er muss die eigene Verhinderung zum Zeitpunkt der Abgabe der fraglichen Äusserung belegen oder Beispiele für die sonstige Nutzung des Accounts durch Dritte nachweisen.

\section{Screenshots als Beweismittel}

33 In gewissen Fällen wird der Arbeitgeber seiner Substantiierungs- und Beweispflicht nur dadurch gerecht werden können, dass er als Beweismittel Screenshots vorlegt, welche die kündigungsrelevanten Inhalte auf dem Netzwerkprofil des Arbeitnehmers belegen. Nicht immer sind diese als Beweise verwertbar.55 Wenn sie in Verletzung des Persönlichkeitsrechts des Arbeitnehmers beschafft worden sind, so muss gemäss Art. 152 Abs. 2 ZPO abgewogen werden: Wiegt die Persönlichkeitsverletzung des Arbeitnehmers oder wiegt das Interesse an der Wahr-

54 ArbG Dessau-Rosslau, Urteil vom 21.2.2012, $1 \mathrm{Ca}$ 148/11.

55 S. dazu den SRF Kassensturzbeitrag vom 16.4.2013, Entlassung wegen Facebook: Der Chef liest mit. 
heitsfindung schwerer?56

34

Hat der Arbeitnehmer die beanstandeten Social Media-Inhalte öffentlich kundgetan, so wird in der Interessenabwägung die Persönlichkeitsverletzung des Arbeitnehmers nicht so schwer wiegen. Ein Beweisverwertungsverbot dürfte dann ausgeschlossen sein. Wenn sich der Arbeitgeber heimlich bzw. mittels Vortäuschens einer falschen Identität Zugang zu ausschliesslich im privaten Kreis hochgeladenen Inhalten verschafft hat, so wird die Beweisverwertung verboten sein. 57

\section{3. Überwachungsmassnahmen}

35 Es gilt $\mathrm{zu}$ beachten, dass Überwachungsmassnahmen sehr strengen Voraussetzungen unterliegen. Immer zulässig sind technische Massnahmen, die nicht personenbezogen ausgewertet werden können. Hingegen sind personenbezogene Kontrollen Datenbearbeitungen, die den strengen Bedingungen von Art. 328b OR und dem Datenschutzgesetz genügen müssen. Gemäss Art. 26 Abs. 1 der Verordnung 3 zum Arbeitsgesetz ist die präventive, vollständige Verhaltensüberwachung eines Arbeitnehmers, z.B. mittels Spyware, grundsätzlich unzulässig. $5^{8}$

56 Weiterführend Yves Rüedi, Rechtswidrig erlangte Beweismittel, in: Annette Dolge (Hrsg.), Substantiieren und Beweisen, Praktische Probleme, Zürich 2013, S. 77 ff.; BGE 131 I 272 E. 3.2.4; vgl. auch BGE 140 III 6.

57 Bauer/Günther (Fn. 29), 67.

58 Vgl. auch Giordano Costa, Internet- und E-MailÜberwachung am Arbeitsplatz, Jusletter 9. Januar 2012; Thomas Geiser, Die Beaufsichtigung des Internetbenutzers im Arbeitsrecht, medialex 4/01, S. 201 ff.; Sylvain Métille, La surveillance électronique des employés, in: Jean-Philippe Dunand/Pascal Mahon (Hrsg.), Internet au travail, Genf 2014, S. 99 ff.; Aubert/Delley (Fn. 3), S. 148 ff., m.w.H.; Holenstein (Fn. 14), S. 94 ff.
36 Das Bundesgericht hatte denn auch in BGE 139 II 7 über den verdeckten Einsatz eines Überwachungsprogrammes zu entscheiden. Die Software sollte der Bestätigung des Verdachts dienen, dass ein Arbeitnehmer die ihm im Informatikbereich zur Verfügung gestellten Mittel für dienstfremde Zwecke missbrauche. Das Bundesgericht befand den Einsatz als unzulässig (Art. 26 Abs. 1 ArGV 3) oder zumindest unverhältnismässig (Art. 26 Abs. 2 ArGV 3). Es hielt das rechtswidrig erlangte Beweismittel für im Prozess nicht verwertbar. Mit der Verneinung der Verwertbarkeit eines auf diese Weise widerrechtlich erlangten Beweismittels fiel auch die Grundlage für eine fristlose Kündigung dahin.

\section{Regelung der Social Media- Nutzung durch den Arbeitgeber}

37 Welche Regelungskompetenzen stehen dem Arbeitgeber für Verhaltensregeln von Arbeitnehmern in sozialen Netzwerken mittels Weisungsrecht gemäss Art. 321d Abs. 1 OR überhaupt zu? Die Nutzung von Benutzerkonten in sozialen Netzwerken gliedert sich aus rechtlicher Sicht wie folgt:59

1. Dienstlich genutzte private Accounts des Arbeitnehmers und Accounts und Profile des Arbeitgebers sind regelbar.

2. Private Accounts mit Verbindung zum Arbeitgeber, wie z.B. LinkedIn, sind durch den Arbeitgeber regelbar, sofern der Arbeitnehmer eine direkte Verbindung zum Arbeitgeber bewusst herstellt. Ein generelles Verbot der Nutzung von berufsbezogenen sozialen Netzwerken durch den Arbeitge-

59 Borsutzky (Fn. 13), S. 648 ff; Dunand (Fn. 3), S. $38 \mathrm{ff}$. 
ber überschreitet den Umfang seines Weisungsrechts. ${ }^{60}$

3. Bei ausschliesslich privat genutzten Accounts wird es nochmals schwieriger. Grundsätzlich können Arbeitgeber Arbeitnehmern die private Nutzung von privaten Social Media Accounts nicht vorschreiben und es besteht keine Regelungsbefugnis des Arbeitgebers. Solange das Verhalten keine Auswirkung auf das Arbeitsverhältnis hat, darf der Arbeitgeber das private Verhalten ausserhalb der Arbeitszeit weder einschränken noch untersagen noch arbeitsrechtlich sanktionieren. Die private Nutzung während der Arbeitszeit muss der Arbeitgeber nicht oder nur beschränkt dulden. Der Arbeitgeber hat ein Interesse daran, dass der Arbeitnehmer keine exzessive private Nutzung des Internets während der Arbeitszeit betreibt, da dieser seine Pflicht zur Leistung von Arbeit gemäss Art. 319 Abs. 1 OR erfüllen soll. Auch die Nutzung unternehmenseigener Infrastruktur kann der Arbeitgeber umfassend regeln und einschränken. ${ }^{61}$ Der Arbeitgeber kann also die Nutzung sozialer Medien nicht nur reglementieren, sondern auf seinen Geräten auch ganz verbieten. Er kann z.B. gewisse Social Media-Internetseiten ganz sperren. ${ }^{62}$ Ein gleichzeitiges Verbot privater Geräte (insbesondere Smartphones) am

6o Kort (Fn. 22), S. $1321 \mathrm{ff}$.

61 Allgemein zum Weisungsrecht des Arbeitgebers, private Nutzung auf betrieblichen Geräten zu verbieten: BGer 4A_430/2008 vom 24.11.2008, E. 4.1, Michèle Stutz/Alexandra Geiger-Steiner, Arbeitsrechtliche Fragen rund um Social Media, Anwaltsrevue 2013, S. 212 ff., S. 214; Egli (Fn. 16), Rz. 33; Dunand (Fn. 3), S. 40; Alder (Fn. 3), S. 277.

62 Stutz/Geiger-Steiner (Fn. 60), S. 214; Egli (Fn. 16), S. 5.
Arbeitsplatz beeinträchtigt das Recht auf Privatleben am Arbeitsplatz (Art. 8 EMRK), weshalb eine solche Beschränkung einer besonderen Rechtfertigung bedarf. ${ }^{63}$

38 Arbeitgebern ist zu empfehlen, ihr Weisungsrecht zur Nutzung von Social Media-Plattformen am Arbeitsplatz mittels Social Media Guidelines wahrzunehmen. ${ }^{64}$ Die Arbeitnehmer sind sich nämlich der Folgen ihres Verhaltens auf Social Media oft nicht bewusst. 65 Deshalb ist es sinnvoll, sie bezüglich des Verhaltens im Netz zu sensibilisieren. ${ }^{66}$ Der Arbeitgeber kann daher Grenzen setzen. Soll die Nutzung sozialer Netzwerke während der Arbeitszeit und über die ITInfrastruktur des Arbeitgebers zulässig sein? In welchem zeitlichen Umfang? Nur für Geschäftszwecke oder auch privat?67

63 Das Recht auf Privatleben leitet der EGMR aus Art. 8 EMRK ab, vgl. dazu folgende Urteile: EGMR, Urteil vom 16.12.1992, Niemietz/Deutschland, Nr. 13710/88; EGMR; Urteil vom 25.6.1997, Halford/Vereinigtes Königreich, Nr. 20605/92; EGMR, Urteil vom 3.7.2007, Copland/Vereinigtes Königreich, Nr. 62617/00; EGMR, Urteil vom 23.9.2010, Schüth/Deutschland, Nr. 1620/03; EGMR, Urteil vom 12.6.2014, Martinez/Spanien, Nr. 56030/07. Kurt Pärli, Schutz der Privatsphäre am Arbeitsplatz in digitalen Zeiten - eine menschenrechtliche Herausforderung, EuZA 2015, S. 58 ff.; vgl. auch BGer 4A_430/2008 vom 24.11.2008 E. 4.1, wonach das Verbot jeglicher privater Tätigkeit am Arbeitsplatz nicht mit der Pflicht des Arbeitgebers, die Persönlichkeit des Arbeitnehmers zu achten und zu schützen (Art. 328 Abs. 1 OR) vereinbar wäre.

64 Dominik Marbet/René Pfister, Soziale Netzwerke (Social Media) - Richtlinien: Worauf Arbeitgeber und Arbeitnehmende achten sollten, Schweizer Arbeitgeber 1/2011, S. 17.

65 Oberwetter (Fn. 48), S. 420; Byers/Mössner (Fn. 45), S. 1666.

66 Paul Melot de Beauregard/Christian Gleich, Social Media am Arbeitsplatz - Chancen und Risiken, DB 2012, S. 2044 ff., S. 2046 f.

67 Vgl. auch den kommentierten Formulierungsvor- 
39 Dabei wirkt sich die Reglementierung unmittelbar auf die Kontrollrechte des Arbeitgebers aus. Ist die Nutzung sozialer Medien am Arbeitsplatz grundsätzlich verboten oder mindestens beschränkt, hat der Arbeitgeber das Recht, die Einhaltung seiner Weisung - unter gewissen datenschutzrechtlichen und arbeitsgesetzlichen Einschränkungen - auch zu kontrollieren. ${ }^{68}$ Ist die Nutzung sozialer Medien hingegen nicht reglementiert, sind Kontrollen nur zulässig, wenn konkrete Hinweise auf eine Verletzung der Treuepflicht durch den Arbeitnehmer bestehen. ${ }^{69}$

40 Die Einführung einer Social MediaRegelung hilft sowohl der Geschäftsleitung als auch den Arbeitnehmern, eine klare Grenze zwischen Dienst und Privatem zu ziehen und Transparenz zu schaffen. Gerade durch Social Media Guidelines kann ein sicherer Umgang der Arbeitnehmer mit sozialen Netzwerken unterstützt werden, was im Ergebnis beiden Seiten zugutekommt.

schlag bei Wolfgang Leist/Maximilian Koschker, Social Media Guidelines: Chancen und Risiken des Mitmach-Wegs im Betrieb verbindlich regeln, BB 2013, S. 2229 ff.; Leitfaden des Eidgenössischen Datenschutz- und Öffentlichkeitsbeauftragten (EDÖB) über Internet- und E-Mail-Überwachung am Arbeitsplatz - Für die Privatwirtschaft, Sept. 2013; Aubert/Delley (Fn. 3), S. 152 f.; Holenstein (Fn. 14), S. 73 ff.

68 Betreffend Überwachungsmassnahmen in engen Grenzen vgl. Leitfaden EDÖB (Fn. 67); BGE 139 III 7.

69 Streiff/von Kaenel/Rudolph (Fn. 10), Art. 328b N 18. 